RAE-IC, Revista de la Asociación Española de Investigación de la Comunicación

vol. 8, núm. 16 (2021), 248-272

ISSN 2341-2690

Recibido el 1 de julio de 2021

DOI: https://doi.org/10.24137/raeic.8.16.12 Aceptado el 1 de septiembre de 2021

\title{
Activismo digital de los movimientos sociales en Portugal y la pandemia Covid-19: el União de Mulheres Alternativa e Resposta y Casa do Brasil de Lisboa
}

Digital activism of social movements in Portugal and the Covid-19 pandemic: the União de Mulheres Alternativa e Resposta and Casa do Brasil de Lisboa

Kraus Luvizotto, Caroline

Universidade Estadual Paulista (UNESP)

caroline.luvizotto@unesp.br

Martin Vicente, Maximiliano

Universidade Estadual Paulista (UNESP)

mm.vicente@unesp.br

Trindade, Ana Carolina

Universidade Estadual Paulista (UNESP)

carolina.trindade@unesp.br

Forma de citar este artículo:

Kraus Luvizotto, C., Martin Vicente, M. y Trindade, A. C. (2021). Activismo digital de los movimientos sociales en Portugal y la pandemia Covid-19: el União de Mulheres Alternativa e Resposta y Casa do Brasil de Lisboa. RAE-IC, Revista de la Asociación Española de Investigación de la Comunicación, 8(16), 248-272. https://doi.org/10.24137/raeic.8.16.12

RAE-IC, Revista de la Asociación Española de Investigación de la Comunicación vol. 8, núm. 16 (2021), 248-272 


\section{Resumen:}

La pandemia COVID-19 ha impuesto una nueva realidad a los movimientos sociales y la participación ciudadana, al prohibir y restringir las protestas sociales y las manifestaciones en las calles, acciones tradicionales de los movimientos sociales. En este escenario se revigora el ciberactivismo o activismo digital, que tiene en la información el principal eje para tomar decisiones. Este artículo analiza el papel de los movimientos sociales durante los primeros meses de la pandemia COVID-19 a partir del contenido informativo del activismo digital de los movimientos sociales portugueses: União de Mulheres Alternativa e Resposta - UMAR y Casa do Brasil de Lisboa - CBL. A partir de la revisión de la literatura y siguiendo los preceptos metodológicos del Análisis de Contenidos, los resultados obtenidos indican que tanto UMAR como CBL ejercieron, fundamentalmente, un activismo digital centrado en redes de solidaridad y de apoyo mutuo, algo que estaba ya establecido en la trayectoria de actuación de cada movimiento. Los movimientos sociales continuaron usando la información para crear y fortalecer lazos, protestar, construir y difundir conocimiento y monitorear las acciones gubernamentales y sus políticas públicas. El reciente episodio de la pandemia mostró que aún quedan muchos desafíos por superar, pero las perspectivas son muy prometedoras para el activismo digital.

Palabras clave: activismo digital, movilización social, información, comunicación digital, pandemia Covid-19, Portugal.

\section{Abstract:}

The Covid-19 pandemic has imposed a new reality on social movements and citizen participation, by prohibiting and restricting social protests and demonstrations in the streets, traditional actions of social movements. In this scenario, cyberactivism or digital activism is revived, which has information as the main axis for making decisions. This article analyzes the role of social movements during the first months of the Covid-19 pandemic based on the informative content of the digital activism of two Portuguese social movements: União de Mulheres Alternativa e Resposta - UMAR and Casa do Brasil 
de Lisboa - CBL. Based on the review of the specialized literature on the subject and following the methodological precepts of Content Analysis, the results obtained indicate that during the period analyzed both UMAR and CBL exercised, fundamentally, a digital activism focused on solidarity and support networks mutual, something that was already established in the trajectory of each movement. Social movements continued to use information to create and strengthen ties, protest, build and disseminate knowledge, and monitor government actions and public policies. The recent episode of the pandemic showed that there are still many challenges to overcome, but the prospects are very promising for digital activism to mobilize people.

Keywords: digital activism, social mobilization, information, digital communication, Covid-19 pandemic, Portugal.

\section{INTRODUCCIÓN}

En la última década, el potencial democratizador de la comunicación digital, sumado a las acciones offline realizadas en las calles, dio visibilidad y voz a innumerables movimientos y activistas sociales. La sociedad global fue marcada por protestas, manifestaciones, marchas y movilizaciones populares en torno de diversas causas sociales. Sin embargo, la pandemia Covid-19 cambió el rumbo de los movimientos sociales a partir de 2020, interrumpiendo abruptamente las protestas en las calles, las marchas, las manifestaciones y las reuniones para debatir las reivindicaciones de los grupos sociales y delinear estrategias de movilización popular. La pandemia Covid-19 introdujo nuevas perspectivas y desafíos para la participación ciudadana, para el desempeño de los movimientos sociales y para el activismo.

La reciente producción científica sobre el tema movimientos sociales y la pandemia Covid-19 abordada en este artículo, indica que existió una fuerte actividad por parte de los movimientos sociales desde el 11 de marzo de 2020, cuando la Organización Mundial de la Salud (OMS) clasificó al coronavirus como pandemia. Los estudios realizados por Pleyers (2020) indican que, a nivel mundial, los movimientos sociales centraron sus acciones en cinco frentes: "protestos (que resurgieron en algunos países a pesar de los 
riesgos para la salud por promover aglomeraciones); defensa de los derechos de los trabajadores; ayuda mutua y solidaridad; monitoreamiento de las acciones gubernamentales y educación y concienciación popular" (Pleyers, 2020, p. 2).

Castells (2013) analiza el desempeño de los movimientos sociales contemporáneos a partir de su conexión multimodal en las redes offline y online. Para Castells los movimientos no dependen de Internet y no encuentran en ella sus causas. Ven a Internet como una posibilidad de conexión, interacción y ruptura que supera las barreras impuestas por el tiempo y el espacio enfrentadas por los movimientos sociales tradicionales. Internet hizo posible el activismo en tiempos de la pandemia Covid-19. En ese contexto la información, uno de los ejes fundamentales para el activismo digital, fortalece las redes de solidaridad y colaboración.

Pleyers (2020) en sus estudios durante los primeros meses de la pandemia identificó cinco formas de actuación de los movimientos sociales. Este artículo analiza la actuación de movimientos sociales durante la pandemia Covid-19 a partir de las informaciones en red elaboradas por dos movimientos sociales en Portugal. Destacamos que los movimientos sociales portugueses elegidos para esta investigación tienen carácter cultural e identitario y representan dos grandes grupos sociales: los inmigrantes, representados aquí por la Casa do Brasil de Lisboa y las mujeres, representadas aquí por la União de Mulheres Alternativa e Resposta - UMAR.

Ambos movimientos concentraron sus acciones exclusivamente en el ambiente online, concretamente, en Facebook. Así, este estudio tiene como objetivo verificar el impacto de la pandemia en los movimientos sociales en el análisis de la agenda de movilización basada en el seguimiento de los movimientos portugueses: UMAR y CBL. Y, concretamente, verificar el contenido informativo producido por el activismo digital de los dos movimientos sociales en el contexto inicial de la pandemia Covid-19. En este escenario, tal período de pandemia podría traer cambios significativos a la sociedad, así como a la participación ciudadana y al desempeño de los movimientos sociales. 
Para analizar los textos de esos dos movimientos publicados en la red, desde el anuncio del estado de emergencia en Portugal, en el día 18 de marzo de 2020, hasta el final del primer gran período de restricciones anunciadas en 30 de junio de 2020, se utilizó la metodología del Análisis del Contenido siguiendo las indicaciones de Laurence Bardin (2011).

A lo largo del artículo, presentamos consideraciones sobre la relación entre los movimientos sociales y la comunicación digital; elaboramos un panorama general sobre los movimientos sociales en tiempos de la pandemia Covid-19; contextualizamos la sociedad portuguesa y los movimientos sociales UMAR y CBL y realizamos el análisis de contenido de sus publicaciones en Facebook.

Después de analizar los resultados obtenidos queda claro que el activismo digital, que tiene como eje principal la información, creció en tiempos de la pandemia Covid-19. Durante el período analizado, tanto la UMAR como la CBL, ejercieron, de manera predominante, un activismo centrado en las acciones de ayuda mutua y solidaridad, lo que está en conformidad con los objetivos y actuaciones históricamente realizadas por esos movimientos.

\section{MARCO TEÓRICO}

\subsection{MOVIMIENTOS SOCIALES Y COMUNICACIÓN DIGITAL}

Los movimientos sociales, aquí entendidos como "acciones sociales colectivas de carácter sociopolítico y cultural que posibilitan distintas formas para que la población se organice y manifieste sus demandas" (Gohn, 2008, p. 13) articulan valores de ciudadanía y participación. Sus acciones pueden manifestarse en forma de protestos, huelgas, manifestaciones, ocupaciones de espacios públicos o privados, y pueden recorrer, o no, a la violencia durante sus acciones. Los movimientos sociales son matrices que generan conocimiento, constituyéndose legítimos portadores de las reivindicaciones sociales (Gohn, 2011). Las redes de comunicación e información que establecen son fundamentales para mantener un espacio público alternativo y construir las bases del 
propio movimiento, lo que nos lleva a entender que la relación entre movimientos sociales, información y comunicación es íntima e intensa (Touraine, 2006).

Estudios recientes indican que internet y los medios digitales posibilitan una nueva comprensión y significado de la participación, de la democracia, del activismo y de las identidades colectivas, pues su estructura permite la articulación de los actores sociales de manera correlacionada. Por esos motivos Internet es considerada uno de los medios de comunicación más democráticos en cuanto al acceso, creación y uso de la información (Castells, 2013; Horn, 2013; Bittencourt, 2014; Peruzzo, 2015; SchererWarren y Luchmann, 2015; Alcântara, 2016; Luvizotto y Cunha, 2020).

El ciberactivismo, o activismo digital, desempeña un papel importante en la lucha por el cambio social (Della Porta y Diani, 2020). Estudios realizados por Volpato, et al. (2019) indican que los movimientos sociales utilizan, simultáneamente, acciones realizadas en la calle (como marchas, actos y protestos) y el activismo digital con recursos de comunicación en tiempo real. De esa forma difunden información sobre sus demandas y su agenda de movilización, al mismo tiempo que legitiman sus causas y presionan a los gobiernos y a los diversos sectores de la sociedad.

A pesar de enfatizar la importancia y el potencial de la comunicación digital para el acceso, la búsqueda y difusión de información de carácter democrático y ciudadano, y también para el desempeño de los movimientos sociales en la época contemporánea (Mattoni, 2017), especialmente en la época de la pandemia del Covid-19, este estudio no ignora las numerosas limitaciones y desafíos relacionados con el acceso a las tecnologías digitales y las particularidades de la comunicación digital. Es segmentada y restringida a poblaciones específicas en algunas regiones periféricas. Por otro lado, es más accesible a la población de países económicamente desarrollados (Gomes, 2018). Lo que se puede afirmar, independiente de tener acceso o no, es que los entornos digitales están dominados actualmente por empresas privadas que tienen como objetivo, sobre todo, lucrar. Es la lógica del capital en sintonía con la racionalidad neoliberal dominante que, de esa manera, transforma y amplía las relaciones capitalistas a nivel global (Dardot y Laval, 2016). Lugar abierto y plural, el ciberespacio 
se convierte en el escenario donde actúan oligopolios mediáticos transnacionales y globales que provocan y negocian relaciones y comportamientos sociales (Dahlgren, 2009). Antes visto como un lugar de desintermediación, el ciberespacio está, en estas primeras décadas del siglo XXI, fuertemente mediado por algoritmos poco transparentes, basados en la recopilación y comercialización de datos personales (Sena, 2020; Cunha, 2015).

Sabiendo de las limitaciones de la comunicación digital y del uso de ella por parte de los movimientos sociales, la siguiente sección del artículo se ter para debate algunas consideraciones generales, perspectivas y desafíos sobre el papel de los movimientos sociales en el contexto de la pandemia Covid-19.

\subsection{LA PANDEMIA COVID-19 Y LOS MOVIMIENTOS SOCIALES}

El primer caso conocido de infección por el nuevo coronavirus SARS-CoV2, se identificó en Wuhan, China, en diciembre de 2019. La infección se propagó rápidamente por el mundo configurándose como una pandemia, según un comunicado de la Organización Mundial de la Salud (OMS), publicado en el día 11 de marzo de $2020^{1}$.

De acuerdo con el Alto Comisionado de las Naciones Unidas para los Derechos Humanos, la superación de la pandemia se centra en la solidaridad y la cooperación, tanto para combatir el virus y mitigar sus efectos nefastos, como para superar los problemas ocasionados a la humanidad. Para que tengan éxito las políticas públicas en el combate a la pandemia es fundamental "el respeto de los derechos humanos en todos los ámbitos, incluyendo los derechos económicos, sociales y culturales, así como los derechos civiles y políticos" (Alto Comisionado de las Naciones Unidas para los Derechos Humanos, 2020, s.p.).

Concordamos con esa línea de pensamiento y defendemos la importancia fundamental de los movimientos sociales y de los activistas que movilizan personas y recursos para

\footnotetext{
${ }^{1}$ Hasta 27 de diciembre de 2020, la pandemia de Covid-19 ya había alcanzado la marca de 80.724.170 infectados y 1.763.714muertes en todo el mundo (OMS).
} 
llevar información y garantizar los derechos de innumerables grupos sociales y poblaciones vulnerables, creando una red online y offline de solidaridad y colaboración.

En el artículo "La pandemia es un campo de batalla. Movimientos sociales en el bloqueo Covid-19", Pleyers (2020) discute la actuación de los movimientos sociales en diferentes países durante el período de restricciones. Identifica que la primera forma de acción de los movimientos sociales, en ese período, está relacionada con las acciones de protesto. Eso sucedió, por ejemplo, en países como Israel, Grecia, Chile, Líbano y Ecuador. Para Pleyers:

La mayoría de los activistas esperaron a que terminaran las restricciones para juntarse con las manifestaciones de protesto. En algunos países los activistas organizaron los movimientos contra las restricciones, a pesar de los riesgos para la salud por juntar aglomeraciones de personas (Pleyers, 2020, p. 4).

La segunda forma de actuación de los movimientos sociales, durante el período de restricciones, se centró en acciones en defensa de los trabajadores. Estas acciones tenían como objetivo ofrecer información y garantizar los derechos no solo de los trabajadores de áreas esenciales que no pudieron parar durante la pandemia, como la salud, el transporte y la producción de alimentos, sino también a los que realizaban teletrabajo, o estaban desempleados (Pleyers, 2020). Esas acciones tuvieron lugar en países como Estados Unidos, Hong Kong, Francia y Bélgica.

Pleyers afirma que la tercera frente de acción de los movimientos sociales durante la pandemia se centró en la ayuda mutua y la solidaridad. "En este período de crisis, los movimientos populares, las organizaciones de base y ciudadanas asumieron un papel de liderazgo en la participación del apoyo mutuo, el apoyo a las necesidades básicas y la solidaridad en su comunidad y fuera de ella" (Pleyers, 2020, p. 5). En todos los continentes los movimientos sociales y las organizaciones de la sociedad civil articularon acciones para ofrecer información sobre la pandemia, sobre la salud física y mental, sobre los derechos civiles y políticos, sobre la seguridad y la prevención de la violencia. Además, establecieron grupos locales de asistencia mutua. Aunque estas acciones ocurrieron a nivel mundial, Pleyers (2020) afirma que fueron más recurrentes en países 
del Sur Global, como en el Brasil, por ejemplo, donde se observaron acciones importantes en la Favela de Paraisópolis y Complexo do Alemão.

"La sociedad civil y los movimientos sociales también actúan como guardianes de las políticas públicas y de los gobiernos" (Pleyers, 2020, p. 7). Desde el inicio de la pandemia Covid-19, movimientos sociales, intelectuales y organizaciones de la sociedad civil han analizado y elaborado informes sobre cómo los gobiernos enfrentaban la crisis sanitaria y social, generando información que servía de apoyo a la participación ciudadana. Según el autor este es el cuarto tipo de actividades que realizan los movimientos sociales durante la pandemia: monitorear las acciones de los gobiernos. Es lo que ocurrió en países como Estados Unidos, países de la Unión Europea y Filipinas, por ejemplo.

La quinta forma de actuación de los movimientos sociales en tiempos de la pandemia Covid-19 enumerada por el autor es la que concentra acciones de educación y sensibilización popular. "La educación y la sensibilización popular son quizás el papel más poderoso de los movimientos sociales, ya que la pandemia se combina con un exceso de información y de noticias falsas y teorías conspiradoras" (Pleyers, 2020, p. 8). Los estudios de Pleyers indican que numerosas redes de movimientos, nacionales e internacionales, están muy involucrados en compartir experiencias, analizar y verificar información a través de plataformas en línea y redes sociales. Nuevamente el autor incluye en esta acción algunos casos brasileños además de citar plataformas digitales que reúnen experiencias internacionales sobre la pandemia.

Este panorama general de las acciones de los movimientos sociales elaborado por Pleyers (2020) nos ofrece condiciones para afirmar que el desempeño de los movimientos sociales es fundamental para mantener las democracias y los derechos civiles en tiempos de crisis. Tomando como punto de partida los estudios realizados por Pleyers (2020), los movimientos sociales portugueses, objetos de estudio de este artículo, se convierten en paradigmas empíricos para comprender su actuación durante la pandemia Covid-19. La siguiente sección presenta el contexto portugués y los movimientos UMAR y CBL. 


\subsection{EL CONTEXTO PORTUGUÉS Y LOS MOVIMIENTOS SOCIALES UNIÃO DE MULHERES} ALTERNATIVA E RESPOSTA (UMAR) Y CASA DO BRASIL DE LISBOA (CBL)

Campos, et al. (2016), realizaron estudios y tipologías sobre los movimientos sociales en la sociedad portuguesa. Los investigadores identificaron seis tipos de movimientos en la actualidad: (1) Nuevos movimientos sociales contra la austeridad: "movimientos que nacen como consecuencia de la crisis económica y financiera, siendo que sus principales reivindicaciones se centran en la lucha contra las medidas de austeridad implementadas por el gobierno". Como ejemplo de esos movimientos los autores citan "Plataforma 15 de Outubro" y "Que se Lixe a Troika"; (2) Movimientos antiglobalización: "predecesores de los nuevos movimientos sociales formados en torno a programas contra la austeridad", como ATTAC Portugal; (3) Nuevos movimientos sociales clásicos: movimientos asociados a causas culturales e identitarias. Como ejemplo mencionan los movimientos LGBT, feministas, inmigración y ambientales; (4) Movimientos radicales que se colocan explícitamente contra el sistema. Incluyen aquí los grupos anarquistas o partidarios de causas anarquistas, "que se caracterizan por prácticas alternativas, de resistencia o que se oponen a los valores de la sociedad dominante"; (5) Movimientos directamente involucrados con el activismo digital: "movimientos, plataformas, grupos u organizaciones que operan principalmente en línea o que utilizan internet y otras tecnologías como principal recurso para defender sus causas". Destacan, Anonymous, el movimiento por el software libre y por el Partido Pirata; y (6) Actores políticos tradicionales: a saber, partidos políticos y sindicatos. "Obviamente, estos no son movimientos sociales, pero tienen una relación de complicidad que se manifiesta de diversas formas" (Campos et al., 2016, p. 34).

La UMAR articula, en la medida de lo posible, su agenda de movilización con las actividades de movimientos feministas, LGBT, negros y culturales. Sus acciones offline han sido constantes y reconocidas por la población portuguesa. El activismo digital cuenta con el apoyo de un equipo que trabaja específicamente con la comunicación digital y las estrategias de movilización popular. El movimiento está institucionalizado y estructurado en formato asociativo. El sitio web oficial 
(http://www.umarfeminismos.org) contiene su trayectoria, registros de acciones realizadas, los proyectos finalizados y los que están en desarrollo, grupos de trabajo y áreas de intervención, además de un call center. La UMAR actua en Lisboa, Oporto, Coimbra, Braga, Viseu, Madeira y las Azores, siendo considerada una de las más importantes representantes del movimiento feminista del país (União de Mulheres Alternativa e Resposta, 2020).

La UMAR está presente en dos redes sociales digitales. Facebook (https://www.facebook.com/UMARfeminismos?ref=hl) tiene un total de $24.084 \mathrm{me}$ gusta y 24.600 seguidores. Publican textos frecuentemente que se alternan entre textos propios, contenido compartido de otros medios de comunicación, videos y fotos de sus acciones. Las publicaciones valoran la agenda de movilización y ofrecen información sobre los derechos de las mujeres. En Instagram (@umar_feminismos), se encuentran registros de sus actividades, pero no es una red social tan activa como Facebook e indica solamente 3.481 seguidores $^{2}$.

La CBL fue fundada en 1992 y se mantiene con la colaboración y apoyo de brasileños, portugueses y otros ciudadanos que simpatizan con la comunidad brasileña. Representante del movimiento inmigrante brasileño en Portugal, su objetivo principal es implementar "políticas públicas, asumiendo un papel fundamental de activismo y de reivindicación de políticas igualitarias para las comunidades inmigrantes en Portugal" (Casa do Brasil de Lisboa, 2020). A pesar de tener como público mayoritario a la comunidad brasileña, la CBL también acoge, ayuda y representa a inmigrantes de cualquier nacionalidad. Su agenda de movilización se centra en tres pilares: intervención social para la existencia de igualdad de oportunidades; activismo con el objetivo de conseguir la igualdad de derechos y la justicia social; y promoción de la interculturalidad e integración de los inmigrantes, entre ellos mismos y con la comunidad portuguesa.

En su sitio web oficial (https://casadobrasildelisboa.pt), se encuentra toda la información relacionada con la historia, acciones, proyectos y convenios del

2 Datos colectados en 27 de diciembre de 2020. 
movimiento. Su activismo para promover la ciudadanía y desde su origen se posicionó contra el racismo, la xenofobia, las desigualdades de género, la persecución de gobiernos a políticos o a partidos. Entre sus acciones destaca la coordinación de las asociaciones de inmigrantes y entidades pro-inmigrantes. Adopta posiciones en el sentido de presionar a las autoridades para que promulguen leyes a favor de los inmigrantes (Casa do Brasil de Lisboa, 2020).

La CBL está presente en dos redes sociales digitales. Facebook (https://www.facebook.com/casadobrasildelisboa/) que tiene 20.755 me gusta y 21.629 seguidores. Publican con frecuencia textos que acostumbran ser extensos y algunos con hashtags. Las interacciones, a través de comentarios, se dan en algunas publicaciones que difunden la agenda de movilización y ofrecen informaciones útiles para los inmigrantes. Instagram (@casadobrasildelisboa) no parece ser una red social digital representativa del movimiento, ya que solo tiene 3.405 seguidores y publicaciones replicadas de Facebook ${ }^{3}$.

La presencia y participación de los movimientos sociales portugueses, en particular la UMAR y la CBL, fueron importantes desde el inicio de la pandemia Covid-19. El primer caso de infección por SARS-CoV2 en Portugal ocurrió en 2 de marzo de 2020. El 18 de marzo, el mismo día que el país recibió la noticia de la segunda muerte causada por el virus, se declaró el estado de emergencia, que persistió hasta el 3 de mayo. A partir de entonces, hasta el 30 de junio, el país comenzó a enfrentar el estado de alerta, en el que fue posible retomar paulatinamente las actividades interrumpidas total o parcialmente durante el estado de emergencia. Hasta esta fecha, todo el territorio portugués tuvo el mismo tratamiento. Por este motivo, nuestro estudio cubre el período del 18 de marzo al 30 de junio de 2020.

El 1 de julio, con el aumento de número de muertes e infectados, Portugal dividió el país en tres niveles. La mayor parte del país se puso en estado de emergencia. En el Área Metropolitana de Lisboa (AML) se decretó el estado de contingencia y en 19 regiones,

${ }^{3}$ Datos colectados en 27 de diciembre de 2020. 
de cinco municipios de la AML, el estado de alerta. El 13 de agosto, el Consejo de Ministros aprobó la prórroga para mantener el estado de contingencia en la región de Lisboa y la situación de alerta en el resto del país. El 9 de noviembre, el Parlamento portugués declaró un nuevo estado de emergencia, en vigor desde el 2 de octubre, con encerramientos nocturnos y confinamiento parcial en casi dos tercios del país. El estado de emergencia, en 20 de noviembre de 2020, se prorrogó por otros 15 días $^{4}$.

En el siguiente apartado, presentamos los procedimientos metodológicos utilizados en este estudio y analizamos el desempeño de la UMAR y la CBL durante el primer mes del estado de emergencia ante la pandemia Covid-19 en Portugal.

\section{METODOLOGÍA}

Después de la etapa de investigación exploratoria, que consistió en una revisión de la bibliografía especializada sobre el tema y de los conceptos que orientan este estudio, realizamos un análisis del contenido de las publicaciones de la UMAR y de la CBL en sus páginas de Facebook. Nuestro objetivo, en esta etapa de la investigación, consistió en verificar el contenido informativo producido por el activismo digital de los dos movimientos sociales en el contexto inicial de la pandemia Covid-19. Y, en seguida, ¿cuál fue el impacto de la pandemia en el activismo digital de los movimientos sociales portugueses elegidos?

El método de Análisis de Contenido, (AC) según Bardin (2011, p. 44), se basa en "un conjunto de técnicas de análisis de la comunicación que utiliza procedimientos sistemáticos y objetivos para describir el contenido de los mensajes". Bardin (2011) señala tres pasos fundamentales para la realización del AC: 1) codificación (elaboración de categorías), 2) categorización (creación de criterios de clasificación) y 3) inferencia (interpretación de los resultados). Nuestra propuesta recopiló publicaciones de forma manual, sin el uso de plataformas de seguimiento, a través de la observación directa,

\footnotetext{
${ }^{4}$ Hasta el 27 de diciembre de 2020, la pandemia Covid-19 ya había alcanzado en Portugal la marca de 394.573 infectados y 6.619 muertes (OMS).
} 
categorizó cada publicación en una tabla de Excel e interpretó los resultados en función de lo recopilado (TRINDADE, 2020).

En la primera etapa del AC, adoptamos como referencia el estudio de Pleyers (2020), considerando como categorías las cinco frentes de actuación de los movimientos sociales durante los primeros meses de la pandemia: a) Acciones de protesta; b) Acciones en defensa de los derechos de los trabajadores; c) Acciones de ayuda mutua y solidaridad; d) Acciones de acompañamiento de las acciones gubernamentales y e) Acciones de educación y sensibilización popular.

En la segunda etapa del AC definimos como período de recolección de datos el mes posterior a la declaración oficial del estado de emergencia ante la pandemia Covid-19 en Portugal. Así, la recolección se llevó a cabo del 18 de marzo al 30 de junio de 2020, período en el que todo el territorio portugués recibió el mismo tratamiento por parte del gobierno central. Por lo tanto, la tabla 1 muestra la cantidad de contenido categorizado y recopilado de las páginas de Facebook de UMAR y CBL:

Tabla 1. Contenidos recopilados de UMAR y CBL

\begin{tabular}{lcc}
\hline \multicolumn{1}{c}{ Categorias } & UMAR & CBL \\
\hline a) Acciones de protesto & 3 & 1 \\
b) Acciones en defensa de los derechos de los trabajadores & 42 & 1 \\
\hline c) Acciones de ayuda mutua y de solidaridad & 179 & 75 \\
d) Acciones de fiscalización de los actos gubernamentales & 13 & 18 \\
e) Acciones de educación popular y de conscienciación & 17 & 11 \\
\hline Total de publicaciones & 254 & 106 \\
\hline
\end{tabular}

Fuente: Elaboración propia (2021).

A partir del 1 de julio, el territorio fue tratado de manera diferente, con más o menos restricciones, según la gravedad de la pandemia en cada región como vimos anteriormente. También definimos que la recolección de datos se realizaría en las páginas oficiales de Facebook de los dos movimientos sociales, ya que estas páginas presentaban información en constante actualización y contenido más representativo sobre la pandemia Covid-19, en comparación con la red social Instagram y el sitio web oficial de cada movimiento. Las publicaciones encontradas mes a mes se muestran en el Gráfico 1: 
Gráfico 1. Publicaciones recopiladas por mes

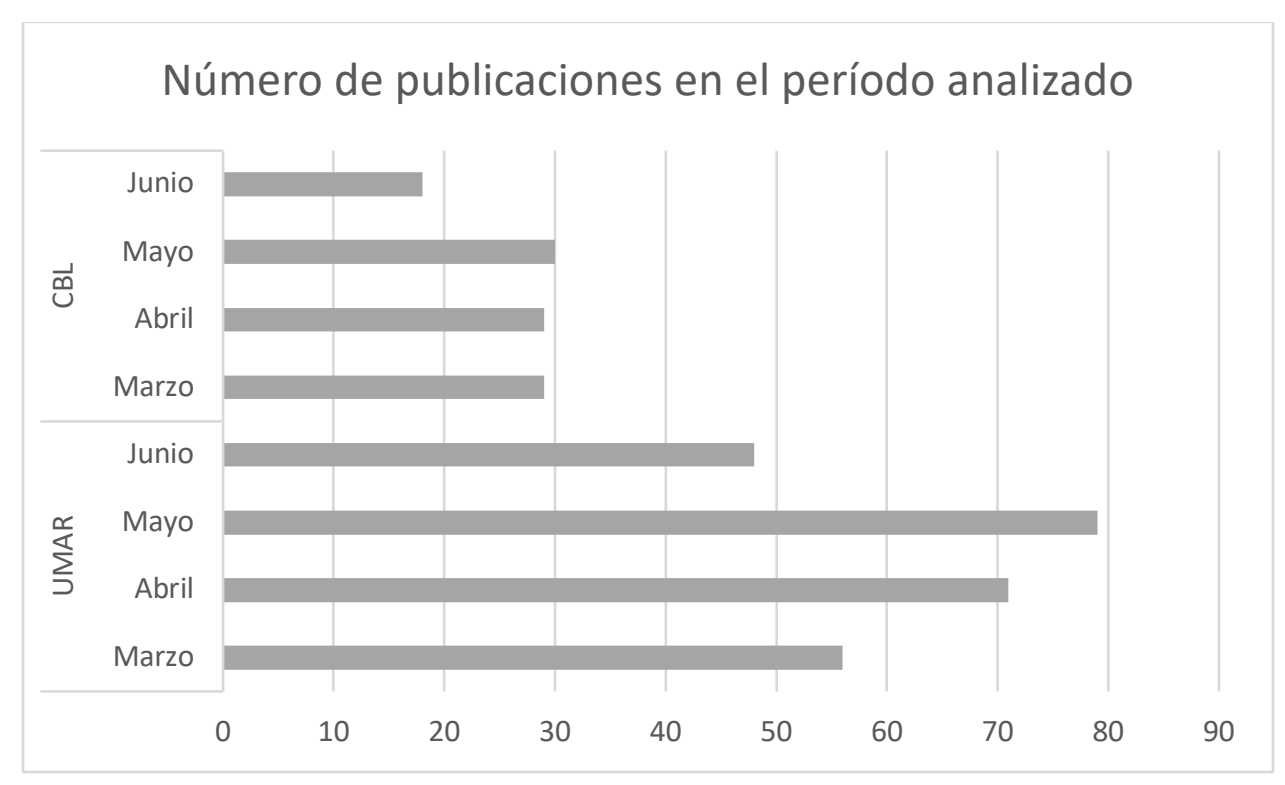

Fuente: Elaboración propia (2021).

Finalmente, después de clasificar las 360 publicaciones, en consonancia con las categorías y criterios establecidos, pasamos a la tercera etapa del AC que consistió en interpretar los resultados con tratamiento cualitativo y cuantitativo.

\section{RESULTADOS}

Durante el período analizado, UMAR realizó 254 publicaciones. Los temas más tratados fueron: formas de distraerse durante la pandemia, apoyo e información para víctimas de violencia doméstica, divulgación de canales de ayuda psicológica gratuita, informaciones sobre la prevención y evolución de la pandemia Covid-19, informaciones sobre las acciones del gobierno portugués y maneras de evitar contagios cotidianamente. Las publicaciones también incluyeron entrevistas, artículos y la divulgación de eventos online relacionados con la temática del movimiento, es decir, el activismo feminista y temas de interés de las mujeres.

Los primeros textos, después de la declaración del estado de emergencia, evidenciaban una preocupación especial de UMAR en manifestar solidaridad con la situación creada por la pandemia. Al mismo tiempo buscaba formas de mantener el bienestar de sus seguidores y de la población portuguesa en general. Así, frecuentemente publicaban lugares donde se ofrecía asistencia psicológica gratuita y cursos online direccionados 
para mejorar la convivencia cotidiana y promover la igualdad de género. La preocupación por precaverse, por un posible crecimiento del número de casos de violencia doméstica, hizo que las publicaciones ofrecieran, además de apoyo psicológico, informaciones sobre formas alternativas de enfrentar la violencia y efectuar las denuncias de malos tratos. También orientaban sobre cómo proceder con las mujeres abusadas o en situación eminente de sufrir violencia doméstica.

La fanpage tiene publicaciones con contenido diversificado y con textos compartidos de otros medios brasileños y portugueses como Folha de São Paulo, El País Brasil, Diário de Notícias, Contacto, Público, DN Madeira, entre otros. En general, estas publicaciones divulgan información sobre el desarrollo de la pandemia Covid-19, tanto en temas de salud como en las acciones tomadas por el gobierno portugués durante el estado de emergencia.

Algunos textos demostraban preocupación de UMAR por la situación de las mujeres trabajadoras, que realizaban teletrabajo, que perdieron su empleo, o que tuvieron que quedarse en casa para cuidar y acompañar a los hijos en las clases impartidas a distancia en un canal de televisión. También se identificaron pasajes preocupados con la inclusión social.

Durante el período analizado, UMAR compartió publicaciones de entidades y movimientos sociales, del gobierno portugués, de las administraciones municipales de diferentes ciudades del país y de los medios de comunicación. También publicó contenido propio dándole voz a activistas del movimiento feminista, personas comunes y especialistas de varias áreas relacionadas con la pandemia, como la salud y el derecho. Desde mayo hasta el final del monitoreo, el 30 de junio, la cantidad de publicaciones relacionadas con contenido activista aumentó significativamente, muchas de ellas relacionadas con el escenario de la pandemia Covid-19.

En función de los contenidos encontrados podemos decir que el activismo digital practicado por UMAR, durante el período estudiado, se puede categorizar de la siguiente manera: categoría a) Acciones de protesto = 3 publicaciones; categoría b) Acciones en defensa de los derechos de los trabajadores = 42 publicaciones; categoría c) Acciones de ayuda mutua y de solidaridad = 179 publicaciones; categoría d) Acciones de fiscalización 
de los actos gubernamentales = 13 publicaciones e categoría e) Acciones de educación popular y de concienciación = 17 publicaciones, totalizando 254 publicaciones.

La clasificación de sus publicaciones, con base en las categorías de acciones de los movimientos sociales durante la pandemia, indica que el $70 \%$ del activismo digital practicado por UMAR se concentró en c) Acciones de ayuda mutua y solidaridad. Destacamos que también se incluyeron en esta categoría, durante el primer mes del estado de emergencia en Portugal, los textos relacionados con el activismo feminista y el universo de la mujer (como género, aborto, arte y cultura), así como todos aquellos que buscaban ofrecer apoyo y solidaridad a mujeres y ciudadanos en general. El segundo tipo de activismo digital más practicado por el movimiento se relaciona con la categoría b) Acciones en defensa de los derechos de los trabajadores, demostrando que, el $17 \%$ de las acciones, estuvieron orientadas, durante la pandemia Covid-19, a ofrecer información y formas de conseguir renta a los trabajadores. d) Fiscalización de las acciones gubernamentales y e) Acciones de educación y sensibilización popular totalizaron, respectivamente, 5\% y 7\%. A lo largo del período analizado, UMAR informó y llamó a la población a participar en tres acciones de protesto offline, fuera de las redes sociales. Esta marca representa el $1 \%$ de las acciones del movimiento y forma parte del activismo digital de tipo a) Acciones de protesto.

Durante el período analizado, CBL publicó 106 textos. La principal preocupación del movimiento, desde que se decretó el estado de emergencia, fue publicar informaciones oficiales del gobierno portugués relacionadas con los inmigrantes para que regularizasen su situación en el Servicio de Extranjeros y Fronteras (SEF). También aparecen textos destinados a apoyar e informar a los brasileños retenidos en Portugal, por la cancelación de vuelos y el cierre de las fronteras aéreas y terrestres, de como podrían proceder. Además, encontramos otros mensajes que ofrecían opciones sobre como distraerse durante la pandemia, sugerencias sobre cómo ayudar a los artistas durante este período (ya que sus lugares de trabajo estaban cerrados), divulgación de canales de ayuda psicológica gratuitos, información sobre prevención del Covid-19 y evolución de la pandemia y noticias de las acciones tomadas por el gobierno portugués 
y la Embajada de Brasil en Portugal. Publicaron noticias del activismo en beneficio de los inmigrantes, uno de los objetivos principales de la CBL.

En cuanto al funcionamiento de CBL, la página de Facebook publicó, a lo largo del mes analizado, información sobre el cierre de la sede y los cambios efectuados en la forma de actuar. La asistencia a los inmigrantes necesitados continuó solo que realizada por Skype y correo electrónico. CBL se comprometió a divulgar información sobre el regreso de brasileños al Brasil compartiendo, desde las redes sociales donde pasó a actuar, la información proporcionada por la Embajada de Brasil sobre vuelos y trámites de las compañías aéreas, incluso poniendo a disposición de sus usuarios los formularios de ANAC (Agencia Nacional de Aviación Civil/BR) para los brasileños que habían cancelado vuelos y tenían dificultades para regresar al Brasil. CBL instruyó a los ciudadanos retenidos en Portugal para que buscasen ayuda en el consulado brasileño en Lisboa, o Porto o Faro.

En abril de 2020, CBL creó el Blog Emergencia Covid-19, con el objetivo de ayudar a la comunidad brasileña y a otros inmigrantes durante el estado de emergencia publicando informaciones y mensajes relacionadas con la salud, vivienda, derechos y trabajo.

A lo largo del período analizado, publicaron textos que divulgaban la situación de los artistas brasileños residentes en Portugal y que buscaban una forma alternativa de sobrevivir durante la pandemia. Con el hashtag \#tempodecomposicao, CBL colocó el nombre del artista, el arte que desarrollaba y una cuenta corriente para transferir cualquier valor económico para quien desease contribuir. Este tipo de publicación sugiere que los inmigrantes brasileños encontraron apoyo en CBL y que recurrieron a ella en situaciones de necesidad.

A partir de las temáticas colocadas en los textos podemos decir que el activismo digital practicado por CBL durante el período analizado se puede categorizar de la siguiente manera: categoría a) Acciones de protesto = 1 publicación; categoría b) Acciones en defensa de los derechos de los trabajadores = 1 publicación; categoría c) Acciones de ayuda mutua y de solidaridad = 75 publicaciones; categoría d) Acciones de fiscalización 
de los actos gubernamentales = 18 publicaciones e categoría e) Acciones de educación popular y de concienciación = 11 publicaciones, totalizando 106 publicaciones.

La clasificación de sus actos, en función de las categorías de acciones de los movimientos sociales durante la pandemia, indica que el $70 \%$ del activismo digital practicado por CBL se concentró en c) Acciones de ayuda mutua y solidaridad. Destacamos que también se incluyeron en esta categoría de actuación los textos relacionados con el activismo y servicios prestados por CBL durante el estado de emergencia, así como todos aquellos que buscaban aportar apoyo y solidaridad a inmigrantes y ciudadanos en general. El segundo tipo de activismo digital más practicado por el movimiento está relacionado con la categoría d) Acciones de fiscalización de los actos gubernamentales, demostrando que el $18 \%$ del activismo tuvo como objetivo dar a conocer las acciones gubernamentales durante el estado de emergencia, principalmente aquellas relacionadas con la situación de los inmigrantes. Destacamos que también se ubicaron en esta categoría los textos relacionados con las acciones de la SEF y la Embajada de Brasil en Portugal, que podemos considerar como activismo digital de tipo b) Acciones en defensa de los derechos de los trabajadores y e) Acciones de educación y sensibilización popular que totalizaron, respectivamente, $1 \%$ y $10 \%$. Durante todo el período analizado, CBL informó e invitó a la gente a participar en una acción de protesta offline, fuera de las redes sociales. Esta marca representa el 1\% de las acciones del movimiento y forma parte del activismo digital de tipo a) Acciones de protesto. La Tabla 2 muestra los porcentajes de contenido encontrados en UMAR y CBL:

Tabla 2. Concentración de contenido encontrado

\begin{tabular}{lcc}
\hline \multicolumn{1}{c}{ Categorias } & UMAR & CBL \\
\hline a) Acciones de protesto & $1 \%$ & $1 \%$ \\
\hline b) Acciones en defensa de los derechos de los trabajadores & $17 \%$ & $1 \%$ \\
\hline c) Acciones de ayuda mutua y de solidaridad & $70 \%$ & $70 \%$ \\
\hline d) Acciones de fiscalización de los actos gubernamentales & $5 \%$ & $18 \%$ \\
\hline e) Acciones de educación popular y de conscienciación & $7 \%$ & $10 \%$ \\
\hline
\end{tabular}

Fuente: Elaboración propia (2021). 
Los números sugieren que durante el primer mes del estado de emergencia en Portugal tanto UMAR como CBL ejercieron, principalmente, un activismo del tipo c) Acciones de ayuda mutua y solidaridad, que está en línea con los objetivos y trayectoria de cada movimiento. Esos actos se basaron, en gran parte, en la información que los movimientos sociales consideraban como fundamentales para ayudar a las personas de los grupos sociales que representaban, en este caso, mujeres e inmigrantes.

Compartimos de la misma opinión de Della Porta (2020, p. 04), y entendemos que los datos recopilados y analizados indican que, en Portugal, así como en todos los países afectados por la pandemia Covid-19, "en vista de las evidentes insuficiencias del Estado y, más aún, del mercado, las organizaciones de los movimientos sociales se constituyen en grupos de apoyo mutuo, promoviendo acciones sociales directas, ayudando a los más necesitados", produciendo resistencias y lazos de solidaridad.

\section{CONCLUSIONES}

Los movimientos sociales surgen o se fortalecen en tiempos de crisis, calamidades o supresión de las libertades individuales y colectivas (Mattoni, 2013). En cuanto al impacto de la pandemia en los movimientos sociales portugueses electos, la enfermedad Covid-19 impuso una nueva situación a los movimientos sociales y a la participación ciudadana, prohibiendo o restringiendo los protestos y manifestaciones en las calles, formas tradicionales de actuación de los movimientos sociales.

En este escenario se destaca el ciberactivismo, o activismo digital, que tiene como principal eje de actuación la información. Ellos la usaron, en tiempos de la pandemia Covid-19, para expresar las reivindicaciones de los movimientos sociales, mostrar su agenda de movilización, llevar ayuda y salvar vidas.

Gracias a las Tecnologías de Información y Comunicación (TIC), los movimientos sociales continuaron usando la información para crear y fortalecer lazos, protestar, luchar por sus pautas, construir y difundir conocimiento y mostrar su proyecto de sociedad. Además, gracias a las TIC, a lo largo de la pandemia Covid-19, los movimientos sociales 
monitorearon las acciones gubernamentales y sus políticas públicas, práctica fundamental para el buen funcionamiento de la democracia.

Comprobamos el impacto ejercido sobre los movimientos sociales en función de la pandemia Covid-19 y del estado de emergencia en Portugal, así como el papel central que ejerció la información en las prácticas del activismo digital. Al observar el activismo digital de UMAR en su página de Facebook, en períodos anteriores al analizado en esta investigación, identificamos contenidos con temáticas relacionadas, en general, con temas de género, igualdad y derechos de las mujeres. Con el inicio del estado de emergencia, UMAR pasó a presentar, de manera intensa, alineamientos relacionados con la prevención y denuncia de la violencia contra las mujeres, ya que el aislamiento social y el confinamiento en casa podrían ocasionar el aumento casos de violencia doméstica.

Observando periodos anteriores al analizado en esta investigación, encontramos que gran parte de los temas abordados en el activismo digital practicado por CBL en Facebook, también proporcionaron información relevante a los inmigrantes sobre su situación legal, o ilegal, en Portugal. Con el establecimiento del estado de emergencia, el movimiento social continuó publicando mensajes esenciales para los inmigrantes, solo que con un enfoque más direccionado sobre la prevención y los impactos causados por la pandemia Covid-19.

Terminamos haciendo referencia a los estudios de Harvey (2020), Della Porta (2020) y Pleyers (2020) cuando sugieren que la crisis provocada por la pandemia Covid-19 debe ser tratada como un momento de ruptura que traerá cambios significativos en nuestras vidas y nuestras sociedades. En este escenario, la participación ciudadana y la actuación de los movimientos sociales es fundamental para garantizar los valores democráticos en las sociedades contemporáneas. Para quienes dudaban del potencial de Internet para la movilización popular y de la importancia de la comunicación digital para el activismo, el reciente episodio de la pandemia Covid-19 mostró que aún quedan muchos desafíos por superar, pero las perspectivas son muy promisorias. 


\section{AGRADECIMIENTOS}

Este artículo presenta los resultados parciales de dos investigaciones financiadas por la Fundação de Amparo à Pesquisa do Estado de São Paulo, Fapesp/Brasil: Processo 19/16693-1 y Processo 19/12815-5.

\section{REFERENCIAS BIBLIOGRÁFICAS}

Alcântara, L. M. (2016). Ciberativismo e a Dimensão Comunicativa dos Movimentos Sociais: repertórios, organização e difusão. Política \& Sociedade, 15(34), 315-332. https://doi.org/10.5007/2175-7984.2016v15n34p315

Alto Comisionado de las Naciones Unidas para los Derechos Humanos (2 de abril de 2020). Covid-19 e a dimensão de direitos humanos. https://bit.ly/37Q7obk

Bardin, L. (2011). Análise de conteúdo. São Paulo: Edições 70.

Bittencourt, M. C. A. (2014). Movimentos sociais e mídia de espalhamento: democratização da comunicação em contexto de convergência. Revista Líbero, 17(33), 51-60. Recuperado de https://bit.ly/3nY63VK

Campos, R., Pereira, I. y Simões, J.A. (2016). Ativismo digital em Portugal: um estudo exploratório. Sociologia, Problemas e Práticas. 82, 27-47. https://doi.org/10.7458/SPP2016826977

Castells, M. (2013). Redes de indignação e esperança: movimentos sociais na era da internet. Rio de Janeiro: Zahar.

Casa do Brasil de Lisboa (2020). O que fazemos - Casa do Brasil de Lisboa. Recuperado de https://bit.ly/3nSysMU

Cunha, I. F. (2015). Da 'desdemocratização' da Europa: democracia, media e corrupção política. Intercom-Revista Brasileira de Ciências da Comunicação, 38(1), 37-63. https://doi.org/10.1590/1809-5844201512 
Dahlgren, P. (2009). Media and Political Engagement: Citizens, Communication, and Democracy. New York: Cambridge University Press.

Dardot, P. y Laval, C. (2016). A nova razão do mundo: ensaio sobre a sociedade neoliberal. São Paulo: Boitempo.

Della Porta, D. (2020, Marzo 26). Movimientos sociales en tiempos de Covid-19: otro mundo es necesario. Open Democracy. Recuperado de https://bit.ly/3pyP8tj

Della Porta, D. y Diani, M. (2020). Organizations and organizing within social movements. En D. Della Porta y M. Diani (2020). Social movements: an introduction (pp. 134-160). Hoboken: Wiley-Blackwell.

Gohn, M.G. (2011). Movimentos sociais na contemporaneidade. Revista Brasileira de Educação, 16(47), 333-361. https://doi.org/10.1590/S1413-24782011000200005

Gohn, M.G. (2008). O protagonismo da sociedade civil - movimentos sociais, ONGs e redes solidárias. São Paulo: Cortez.

Gomes, W. (2018). A democracia no mundo digital: histórias, problemas e temas. São Paulo: Edições Sesc SP.

Harvey, D. (2020). Anti-Capitalist Politics in the Time of Covid-19. Retrieved from Global University. Recuperado de https://bit.ly/34LC8Z8

Horn, J. (2013). Gender and social movements: Overview report. Brighton, England: Institute of Development Studies.

Luvizotto, C. K. y Cunha, I. F. (2020). Mídia, Regulação e Movimentos Sociais em Portugal. En C. K. Luvizotto y I.F. Cunha. (Eds.). Comunicação, cidadania e movimentos sociais: Perspectivas contemporâneas da participação cidadã (pp. 37-65). Aveiro: Ria Editorial. Recuperado de https://bit.ly/2M1FOPV

Luvizotto, C. K., Napolitano, C. J. y Trindade, A. C. (2020). A informação sobre a Democratização da Comunicação no blog do Intervozes na Revista da Carta Capital. 
Revista Latinoamericana de Ciencias de la Comunicación, 19(34), 38-48. Recuperado de https://bit.ly/3n2ST8I

Mattoni, A. (2017). At the intersections of journalisms and politics. Semi-established media and social movements in Italy. Parágrafo, 5(2), 78-93. Recuperado de https://bit.ly/38Rd6JA

Mattoni, A. (2013). Repertoires of communication in social movement processes. En B. Cammaerts, A. Mattoni y P. Mccurdy (Eds.). Mediation and protest movements (pp. 3956). Bristol: Intellect.

Organização Mundial da Saúde. Surto de doença por coronavírus (COVID-19). Recuperado de https://bit.ly/3nZOM03

Peruzzo, C. (2015). Representações dos movimentos populares na mídia e como eles se representam: visibilidade pública e perspectivas cívicas. Conexão-Comunicação e Cultura, 14(28), 31-49. Recuperado de https://bit.ly/37TKP5H

Pleyers, G. (2020). The Pandemic is a battlefield. Social movements in the Covid-19 lockdown. Journal of Civil Society, 16(4), 1-18.

https://doi.org/10.1080/17448689.2020.1794398

Scherer-Warren, I. y Luchmann, L. (2015). Um panorama dos estudos sobre os movimentos sociais e a participação no Brasil. En I. Scherer-Warren y L. Luchmann (Eds). Movimentos sociais e engajamento político: trajetórias e tendências analíticas. Florianópolis: Ed. UFSC.

Sena, K. (2020). Comunicação Pública e Redes Digitais - Atores, Técnicas e Políticas (Tese de Doutorado). Universidade Estadual Paulista, Bauru, Brasil. Recuperado de https://repositorio.unesp.br/handle/11449/194513

Touraine, A. (2006). Na fronteira dos movimentos sociais. Sociedade e Estado, 21(1), 17-28. https://doi.org/10.1590/S0102-69922006000100003 
Trindade, A. C. (2020). O potencial das fanpages dos movimentos sociais em prol da democratização da comunicação: um estudo sobre a comunicação online do Intervozes e Fórum Nacional pela Democratização da Comunicação. Dissertação de Mestrado, Universidade Estadual Paulista Júlio de Mesquita Filho, Bauru, SP, Brasil. União de Mulheres Alternativa e Resposta (2020). Quem somos - União de Mulheres Alternativa e Resposta (UMAR). Recuperado de https://bit.ly/3nToURJ

Volpato, A. N., Luvizotto, C. K. y Versuti, C. D. (2019). Visibilidade Como Estratégia, Estratégias de Visibilidade: Movimentos sociais contemporâneos na internet. Revista ECO-Pós, 22(1), 352-383. https://doi.org/10.29146/eco-pos.v22i1.15992 